\title{
A useful reparameterisation to obtain samples from conditional inverse Wishart distributions
}

\author{
Inge Riis Korsgaard ${ }^{\mathrm{a} *}$, Anders Holst Andersen ${ }^{\mathrm{b}}$, \\ Daniel Sorensen ${ }^{\mathrm{a}}$ \\ a Department of Animal Breeding and Genetics, Research Centre Foulum, \\ PO Box 50, DK-8830 Tjele, Denmark \\ b Department of Theoretical Statistics, University of Aarhus, \\ DK-8000 Aarhus C, Denmark
}

(Received 12 December 1997; accepted 6 January 1999)

\begin{abstract}
A Bayesian joint analysis of normally distributed traits and binary traits, using the Gibbs sampler, requires the drawing of samples from a conditional inverse Wishart distribution. This is the fully conditional posterior distribution of the residual covariance matrix of the normally distributed traits and liabilities of the binary traits. Obtaining samples from the conditional inverse Wishart distribution is not straightforward. However, combining well-known matrix results and properties of the Wishart distribution, it is shown that this can be easily carried out by successively drawing from Wishart and normally distributed random variables. (c) Inra/Elsevier, Paris
\end{abstract}

conditional inverse Wishart distribution / Gibbs sampling / binary traits / residual covariance matrix

Résumé - Reparamétrisation permettant d'obtenir des échantillons tirés d'une loi de Wishart inverse conditionnée. Une analyse bayésienne utilisant l'échantillonnage de Gibbs, de caractères distribués normalement conjointement avec des caractères binaires, requiert le tirage d'échantillons dans une loi de Wishart inverse conditionnée. Il s'agit de la distribution a posteriori de la matrice de covariance résiduelle des caractères distribués normalement et des variables latentes correspondant aux variables binaires. L'obtention d'échantillons correspondants n'est pas évidente. Cependant l'utilisation de résultats bien connus sur les matrices et des propriétés de la distribution de Wishart permet d'aboutir à une solution en tirant

\footnotetext{
* Correspondence and reprints

E-mail: snfirk@genetics.sh.dk or IngeR.Korsgaard@agrsci.dk
} 
successivement dans une loi de Wishart et dans des lois gaussiennes. (C) Inra/Elsevier, Paris

distribution de Wishart inverse conditionnée / échantillonnage de Gibbs / caractères binaires / matrice de covariance résiduelle

\section{INTRODUCTION}

Markov chain Monte Carlo makes possible the exploration of posterior distributions with relative ease, using models which are computationally too complex to be implemented with other approaches. A case in point is the models for a joint analysis of a normally distributed trait (such as weight gain or yield of milk) and a binary trait (resistant or not resistant to disease, twin or single birth in cattle) - where the binary trait is modelled via the threshold model [9], which invokes the existence of an unknown continuously distributed underlying variable, the liability. A Bayesian analysis of such traits, using the Gibbs sampler, requires the drawing of samples from a conditional inverse Wishart distribution (e.g. $[3,5,8])$. This is the fully conditional posterior distribution of the residual covariance matrix of the normally distributed traits and liabilities of the binary traits. Obtaining samples from the conditional inverse Wishart distribution is not straightforward.

The purpose of this note is to present an easy method to obtain samples from the conditional inverse Wishart distribution, where the conditioning is on a block diagonal submatrix, $\mathbf{R}_{22}$, equal to the identity matrix of the inverse Wishart distributed matrix, $\mathbf{R}=\left(\begin{array}{ll}\mathbf{R}_{11} & \mathbf{R}_{12} \\ \mathbf{R}_{21} & \mathbf{R}_{22}\end{array}\right)$. This is carried out by combining well-known relationships between a partitioned matrix and its inverse and properties of Wishart distributions. The proposed method can alternatively be arrived at by using both another reparameterisation and the properties of the inverse Wishart distribution. This was carried out in Drèze and Richard [2] and is well-known in the econometric literature. The need for sampling from a conditional inverse Wishart distribution is motivated by a Bayesian multivariate analysis of $p_{1}$ normally distributed traits and $p_{2}$ binary traits, $p_{1}, p_{2} \geq 1$, using the Gibbs sampler and data augmentation.

\section{THE MODEL}

Assume that $p_{1}$ normally distributed traits and $p_{2}$ traits with binary response are observed for each animal. Data on animal $i$ are $\mathbf{y}_{i}=\left(\mathbf{y}_{i 1}^{\prime}, \mathbf{y}_{i 2}^{\prime}\right)^{\prime}$, where $y_{i 1 j}$ is the observed value of the $j$ th normally distributed trait, $j=$ $1, \ldots, p_{1}$, and $y_{i 2 k}$ is the observed value of the $k$ th binary trait, $k=1, \ldots, p_{2}$. It is assumed that the outcome of $Y_{i 2 k}$ is determined by an underlying continuous random variable, the liability, $U_{i k}$, where $Y_{i 2 k}=1$ if $U_{i k} \geq \tau$ and $Y_{i 2 k}=0$ if $U_{i k}<\tau$, where $\tau$ is a fixed threshold, often assumed to be equal to zero. Let $\mathbf{W}_{i}=\left(\mathbf{Y}_{i 1}^{\prime}, \mathbf{U}_{i}^{\prime}\right)^{\prime}$ and define $\mathbf{W}$ as the $n p$-dimensional column vector, containing the $\mathbf{W}_{i} s, \mathbf{W}^{\prime}=\left(\mathbf{W}_{1}^{\prime}, \ldots, \mathbf{W}_{N}^{\prime}\right), p=p_{1}+p_{2}$. It is assumed that

$$
\mathbf{W} \mid \mathbf{b}, \mathbf{a}, \mathbf{R}=\mathbf{r}, \mathbf{R}_{22}=\mathbf{I}_{p_{2}} \sim N_{n p}\left(\mathbf{X b}+\mathbf{Z a}, \mathbf{I}_{n} \otimes\left(\begin{array}{ll}
\mathbf{r}_{11} & \mathbf{r}_{12} \\
\mathbf{r}_{21} & \mathbf{I}_{p_{2}}
\end{array}\right)\right)
$$


where $\mathbf{X}$ and $\mathbf{Z}$ are design matrices associating $\mathbf{W}$ with 'fixed' effects, $\mathbf{b}$, and additive genetic values, a, respectively. The usual condition, $\left(\mathbf{R}_{22}\right)_{k k}=1$ (e.g. [1]), has been imposed in the conditional probit model for $Y_{i 2 k}$ given a, $k=1, \ldots, p_{2}$. Furthermore it is assumed that liabilities of the binary traits are conditionally independent, given $\mathbf{b}$ and $\mathbf{a}$. The following prior distributions are assumed: $\mathbf{b}$ is uniform,

$$
\mathbf{a} \mid \mathbf{G}=\mathbf{g} \sim N_{q p}(\mathbf{0}, \mathbf{A} \otimes \mathbf{g}), \mathbf{G} \sim I W_{p}\left(\Sigma_{G}, f_{G}\right)
$$

and that $\mathbf{R} \mid \mathbf{R}_{22}=\mathbf{I}_{p_{2}}$ follows a conditional inverse Wishart distribution with density up to proportionality given by:

$$
\left|\left(\begin{array}{ll}
\mathbf{r}_{11} & \mathbf{r}_{12} \\
\mathbf{r}_{21} & \mathbf{I}_{p_{2}}
\end{array}\right)\right|^{-\left(f_{R}+p+1\right) / 2} \exp \left\{-\frac{1}{2} \operatorname{tr}\left(\Sigma_{R}^{-1}\left(\begin{array}{ll}
\mathbf{r}_{11} & \mathbf{r}_{12} \\
\mathbf{r}_{21} & \mathbf{I}_{p_{2}}
\end{array}\right)^{-1}\right)\right\}
$$

Augmenting with the vector $\mathbf{U}=\left(\mathbf{U}_{1}, \ldots, \mathbf{U}_{n}\right)^{\prime}$ of liabilities, and also assuming that a priori $\mathbf{b},(\mathbf{a}, \mathbf{G})$ and $\mathbf{R}$ are mutually independent, it follows (e.g. [5]), that the fully conditional posterior distributions required to implement the Gibbs sampler are easy to sample from with the exception of the fully conditional posterior distribution of $\mathbf{R} \mid \mathbf{R}_{22}=\mathbf{I}_{p_{2}}$. The fully conditional posterior distribution of $\mathbf{R} \mid \mathbf{R}_{22}=\mathbf{I}_{p_{2}}$ is conditional inverse Wishart distributed with density proportional to equation (2) with $\Sigma_{R}$ replaced by $\Lambda=\left(\Sigma_{R}^{-1}+\sum_{i=1}^{n}\left(\mathbf{w}_{i}-\mathbf{x}_{i} \mathbf{b}-\mathbf{z}_{i} \mathbf{a}\right)\left(\mathbf{w}_{i}-\mathbf{x}_{i} \mathbf{b}-\mathbf{z}_{i} \mathbf{a}\right)^{\prime}\right)^{-1}$ and with degrees of freedom $f_{R}$ replaced by $f_{R}+n$. In the method to be proposed for sampling from equation (2) in a computationally simple manner, the properties summarised below are essential.

Assume that $\mathbf{R} \sim I W_{p}(\Sigma, f)$ and let $\mathbf{V}=\mathbf{R}^{-1}$, then $\mathbf{V} \sim W_{p}(\Sigma, f)$. Furthermore, define $\mathbf{T}=\left(\mathbf{T}_{1}, \mathbf{T}_{2}, \mathbf{T}_{3}\right)$ by $\mathbf{T}_{1}=\mathbf{V}_{11}, \mathbf{T}_{2}=\mathbf{V}_{11}^{-1} \mathbf{V}_{12}$, and $\mathbf{T}_{3}=\mathbf{V}_{22.1}$ $=\mathbf{V}_{22}-\mathbf{V}_{21} \mathbf{V}_{11}^{-1} \mathbf{V}_{12}$, where $\mathbf{V}=\left(\begin{array}{ll}\mathbf{V}_{11} & \mathbf{V}_{12} \\ \mathbf{V}_{21} & \mathbf{V}_{22}\end{array}\right)$ is a partitioning of $\mathbf{V} ; \mathbf{V}_{11}$ is $p_{1} \times p_{1}$ and $\mathbf{V}_{22}$ is $p_{2} \times p_{2}$. Then the following results hold:

Result 1: there is a one to one relationship between $\mathbf{T}$ and $\mathbf{R}$ given by

$$
\mathbf{T}_{1}=\mathbf{R}_{11.2}^{-1}=\left(\mathbf{R}_{11}-\mathbf{R}_{12} \mathbf{R}_{22}^{-1} \mathbf{R}_{21}\right)^{-1}, \mathbf{T}_{2}=-\mathbf{R}_{12} \mathbf{R}_{22}^{-1} \text { and } \mathbf{T}_{3}=\mathbf{R}_{22}^{-1}
$$

and $\quad \mathbf{R}_{11}=\mathbf{T}_{1}^{-1}+\mathbf{T}_{2} \mathbf{T}_{3}^{-1} \mathbf{T}_{2}^{\prime}, \mathbf{R}_{12}=-\mathbf{T}_{2} \mathbf{T}_{3}^{-1}$ and $\mathbf{R}_{22}=\mathbf{T}_{3}^{-1}$

Result 2: $\mathbf{T}_{1} \sim W_{p_{1}}\left(\Sigma_{11}, f\right)$

Result 3: $\mathbf{T}_{2} \mid \mathbf{T}_{1}=\mathbf{t}_{1} \sim N_{p_{1} \times p_{2}}\left(\Sigma_{11}^{-1} \Sigma_{12}, \mathbf{t}_{1}^{-1} \otimes \Sigma_{22.1}\right)$, where $\Sigma_{22.1}=\Sigma_{22}$ $-\Sigma_{21} \Sigma_{11}^{-1} \Sigma_{12}$

Result 4: $\mathbf{T}_{3} \sim W_{p_{2}}\left(\Sigma_{22.1}, f-p_{1}\right)$

Result 5: $\left(\mathbf{T}_{1}, \mathbf{T}_{2}\right)$ is independent of $\mathbf{T}_{3}$, which implies that the conditional distribution of $\left(\mathbf{T}_{1}, \mathbf{T}_{2}\right)$ given $\mathbf{T}_{3}=\mathbf{t}_{3}$ is equal to the marginal distribution of $\left(\mathbf{T}_{1}, \mathbf{T}_{2}\right)$

Result 6: $\left(\mathbf{R}_{22}=\mathbf{I}_{p_{2}}\right) \Leftrightarrow\left(\mathbf{T}_{3}=\mathbf{I}_{p_{2}}\right)$

Result 1 is immediate. Results 2, 4 and 5 all can be found in Mardia et al. [7] and result 3 in Lauritzen [6]. Result 6 follows from result 1. 


\section{AN ALTERNATIVE PARAMETERISATION}

Let $\mathbf{R} \sim I W_{p}\left(\Sigma, f_{R}+n\right)$ be reparameterised in terms of $\left(\mathbf{T}_{1}, \mathbf{T}_{2}, \mathbf{T}_{3}\right)$ given by result 1 :

$$
\mathbf{R}=\left(\begin{array}{cc}
\mathbf{T}_{1}^{-1}+\mathbf{T}_{2} \mathbf{T}_{3}^{-1} \mathbf{T}_{2}^{\prime} & -\mathbf{T}_{2} \mathbf{T}_{3}^{\prime} \\
-\mathbf{T}_{3}^{-1} \mathbf{T}_{2}^{\prime} & \mathbf{T}_{3}^{-1}
\end{array}\right)
$$

with the distribution of $\left(\mathbf{T}_{1}, \mathbf{T}_{2}, \mathbf{T}_{3}\right)$ as specified in results $2,3,4$ and 5 .

The distribution of $\mathbf{R} \mid\left(\mathbf{R}_{22}=\mathbf{I}_{p_{2}}\right)$ is that of $\mathbf{R} \mid\left(\mathbf{T}_{3}=\mathbf{I}_{p_{2}}\right)$. This follows because $\mathbf{T}_{3}=\mathbf{R}_{22}^{-1}$ is a one to one transformation of $\mathbf{R}_{22}$ (property (10.4.3) from calculus of conditional distributions in Hoffmann-Jørgensen [4]) and because of result 6. Next inserting $\mathbf{T}_{3}=\mathbf{I}_{p_{2}}$ in $\mathbf{R}$ (property (10.4.4) in Hoffmann-Jørgensen [4]) it follows from result 5 that the distribution of $\mathbf{R} \mid\left(\mathbf{T}_{3}=\mathbf{I}_{p_{2}}\right)$ is that of

$$
\left(\begin{array}{cc}
\mathbf{T}_{1}^{-1}+\mathbf{T}_{2} \mathbf{T}_{2}^{\prime} & -\mathbf{T}_{2} \\
-\mathbf{T}_{2}^{\prime} & \mathbf{I}_{p_{2}}
\end{array}\right)
$$

From above it follows that if $\mathbf{t}_{1}$ is sampled from $\mathbf{T}_{1} \sim W_{p_{1}}\left(\Sigma_{11}, f_{R}+n\right)$, next $\mathbf{t}_{2}$ from $\mathbf{T}_{2} \mid \mathbf{T}_{1}=\mathbf{t}_{1} \sim N_{p_{1} \times p_{2}}\left(\Sigma_{11}^{-1} \Sigma_{12}, \mathbf{t}_{1}^{-1} \otimes \Sigma_{22.1}\right)$, then

$$
\mathbf{r}=\left(\begin{array}{cc}
\mathbf{t}_{1}^{-1}+\mathbf{t}_{2} \mathbf{t}_{2}^{\prime} & -\mathbf{t}_{2} \\
-\mathbf{t}_{2}^{\prime} & \mathbf{I}_{p_{2}}
\end{array}\right)
$$

is a realised matrix from the conditional inverse Wishart distribution of $\mathbf{R}$ given $\mathbf{R}_{22}=\mathbf{I}_{p_{2}}$.

\section{CONCLUSION}

We have presented a simple method to draw samples from conditional inverse Wishart distributions. The conditioning is on $\mathbf{R}_{22}$ equal to the identity matrix, where $\mathbf{R}=\left(\begin{array}{ll}\mathbf{R}_{11} & \mathbf{R}_{12} \\ \mathbf{R}_{21} & \mathbf{R}_{22}\end{array}\right)$ is a partitioning of an inverse Wishart distributed matrix. The method is relevant in a Bayesian joint analysis of normally distributed and binary traits (the latter with associated liabilities), using the Gibbs sampler. The methodology was illustrated based on models with additive genetic effects only. The generalisation to several random effects is immediate.

\section{ACKNOWLEDGEMENT}

The authors would like to thank a referee for useful comments and suggestions. 


\section{REFERENCES} 1989.

[1] Cox D.R., Snell E.J., Analysis of Binary Data, Chapman and Hall, London,

[2] Drèze J.H., Richard J.-F., Bayesian analysis of simultaneous equation systems, in: Griliches Z., Intriligator M.D. (Eds.), Handbook of Econometrics, North-Holland Publishing Company, vol. 1, 1983, pp. 587-588.

[3] Jensen J., Bayesian analysis of bivariate mixed models with one continuous and one binary trait using the Gibbs sampler, Proceedings of the 5th World Congress on Genetics Applied to Livestock Production 18 (1994) 333-336.

[4] Hoffmann-Jørgensen J., Probability with a View toward Statistics, Chapman and Hall, New York, 1994.

[5] Korsgaard I.R., Genetic analysis of survival data, Ph.D. thesis, University of Aarhus, Denmark, 1997.

[6] Lauritzen S.L., Graphical Models, Oxford University Press, New York, 1996.

[7] Mardia K.V., Kent J.T., Bibby J.M., Multivariate Analysis, Academic Press, Great Britain, 1979.

[8] Sorensen D., Gibbs sampling in quantitative genetics, Internal report no. 82 from the Danish Institute of Animal Science, 1996.

[9] Wright S., An analysis of variability in number of digits in an inbred strain of guinea pigs, Genetics 19 (1934) 506-536. 